\title{
Investigating Optical Properties of One-Dimensional Photonic Crystals Containing Semiconductor Quantum Wells
}

\author{
Mahshid Mokhtarnejad, ${ }^{1}$ Morteza Asgari, ${ }^{2}$ and Arash Sabatyan ${ }^{3}$ \\ ${ }^{1}$ Atomic and Molecular Physics Department, Faculty of Physics, University of Tabriz, Tabriz, Iran \\ ${ }^{2}$ Mechanical Engineering Department, Sharif University of Technology, Tehran, Iran \\ ${ }^{3}$ Physics Department, Faculty of Sciences, Urmia University, Urmia, Iran \\ Correspondence should be addressed to Mahshid Mokhtarnejad; mahshid.mokhtarnejad@gmail.com
}

Received 21 October 2016; Revised 8 February 2017; Accepted 5 April 2017; Published 13 June 2017

Academic Editor: Giulio Cerullo

Copyright (C) 2017 Mahshid Mokhtarnejad et al. This is an open access article distributed under the Creative Commons Attribution License, which permits unrestricted use, distribution, and reproduction in any medium, provided the original work is properly cited.

\begin{abstract}
This study examined MQWs made of InGaAs/GaAs, InAlAs/InP, and InGaAs/InP in terms of their band structure and reflectivity. We also demonstrated that the reflectivity of MQWs under normal incident was at maximum, while both using a strong pump and changing incident angle reduced it. Reflectivity of the structure for a weak probe pulse depends on polarization, intensity of the pump pulse, and delay between the probe pulse and the pump pulse. So this system can be used as an ultrafast all-optical switch which is inspected by the transfer matrix method. After studying the band structure of the one-dimensional photonic crystal, the optical stark effect (OSE) was considered on it. Due to the OSE on virtual exciton levels, the switching time can be in the order of picoseconds. Moreover, it is demonstrated that, by introducing errors in width of barrier and well as well as by inserting defect, the reflectivity is reduced. Thus, by employing the mechanism of stark effect MQWs band-gaps can be easily controlled which is useful in designing MWQ based optical switches and filters. By comparing the results, we observe that the reflectivity of MWQ containing 200 periods of InAlAs/InP quantum wells shows the maximum reflectivity of $96 \%$.
\end{abstract}

\section{Introduction}

Photonic crystals (PCs), which are artificially fabricated materials with periodic structures, have high importance due to their capability to create forbidden frequencies known as photonic band-gaps (PBGs) [1]. PBGs are widely used in optical reactors [2], photon localization [3], spontaneous emission control from atoms [4,5], PC waveguides fabrication [6], and so on. Alongside the geometrical parameters of the PCs, the constituent materials types used in the PC have a great impact on their band structure. Optical properties of the PCs of various types of materials such as metals, semiconductors, graphenes, and metamaterials are explored in previous studies [7-10]. However, PBGs in conventional dielectric PCs strongly depend on the polarization and incidence angle of the electromagnetic waves and this may differ in other types of PCs as well.

On the other hand, reflection of PBGs is an essential feature for controlling the reflecting spectrum of a PC, which can be provided by regulation of optical properties for constituent materials of a photonic crystal. Due to limited rate for conventional electric driven switches and need for enhancement of network communication capacity, it is necessary to use all-optical devices instead of electrons to speed up data transferring and processing [11, 12].

In this article, optical properties and reflectivity of a signal which is in resonance with an active band-gap of the one-dimensional resonant photonic crystal containing InGaAs/GaAs, InAlAs/InP, and InGaAs/InP semiconductor multiple quantum wells (MQWs) are investigated. Maxwell's equations, the continuity of the tangential component of the electromagnetic field, and transfer matrix method were applied to study the role of incident angle and photonic band-gap of InGaAs/GaAs, InAlAs/InP, and InGaAs/InP that are in resonance with the excitonic level in the presence and absence of a strong pump pulse. Unlike conventional passive photonic band-gap, when excitons in quantum wells with Bragg frequency are affected by strong optical pump 


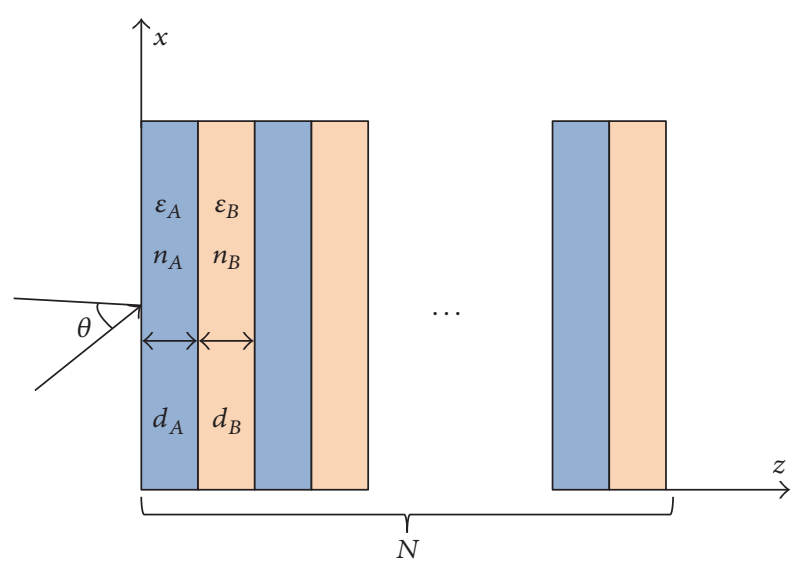

FIGURE 1: A schematic of 1D photonic crystal containing $N$ multiple quantum wells with different refractive index. $d_{B}$ is the width of quantum well and $d_{A}$ is width of quantum barrier which in the following will be shown with $d_{w}$ and $d_{b}$, respectively.

pulse at a frequency close to the frequency of exciton, it is expected that the position and width of photonic band-gap modulated through the Stark effect and the reflectivity to not be in resonance with the exciton anymore. Consequently, this might help to control the band-gap of photonic crystals by use of a pulse pump along with the Stark effect $[13,14]$.

\section{Mathematical Approach}

1D multiple quantum well PCs with a periodic structure of $(A B)^{N}$ were considered as shown in Figure 1, where $A$ and $B$ represent PC consisting of two different dielectric materials with the refractive indices of $n_{A}, n_{B}$ and thicknesses of $d_{A}$ and $d_{B} . N$ also denotes the number of QW periods. Since the layers are nonmagnetic, their permeabilites are $\mu_{A}=\mu_{B}=1$.

The transfer matrix approach was used in order to investigate the band-gap of semiconductor MQWs [15]. Suppose that an electric wave (E), along the $y$-direction, is normally (along the $z$-direction) incident on the structure as shown in Figure 1 . The structure is surrounded by air. In general, the electric and magnetic fields at any two positions $z$ and $z+\Delta z$ in the same layer can be related via a transfer matrix. The transfer matrix of the primitive cell of a 1D photonic crystal can be written [16]:

$$
M_{j}^{(\alpha)}=M_{b}^{1 / 2} M_{b w} M_{w} M_{b w}^{-1} M_{b}^{1 / 2},
$$

where

$$
M_{b}^{1 / 2}=\left(\begin{array}{cc}
e^{i \varphi_{b} / 2} & 0 \\
0 & e^{-i \varphi_{b} / 2}
\end{array}\right)
$$

is the transfer matrix through the halves of barriers which surround the entire quantum well and $\varphi_{b}=\omega n_{b} d_{b} \cos \theta_{b} / c$ is the phase increment of the light propagation through the barrier. Furthermore, $n_{b}, d_{b}, \theta_{b}$, and $c$ are the refractive index of barrier, barrier width, incident angle, and the vacuum velocity through the barrier, respectively. Considering normal illumination, $\theta_{b}$ should be equal to $0^{\circ}$. The electromagnetic wave through the interface between the well and barrier of QW could be described by

$$
M_{b w}=M_{w b}^{-1}=\frac{1}{1+\varrho}\left(\begin{array}{ll}
1 & \varrho \\
\varrho & 1
\end{array}\right),
$$

where $\varrho=\left(n_{w}-n_{b}\right) /\left(n_{w}+n_{b}\right)$ is the Fresnel reflection coefficient.

Transfer matrix of quantum well is

$$
M_{w}=\left(\begin{array}{cc}
(1-i \zeta) e^{i \varphi_{w}} & -i \zeta \\
i \zeta & (1+i \zeta) e^{-i \varphi_{w}}
\end{array}\right),
$$

where $\varphi_{w}=\omega n_{\omega} d_{\omega} \cos \theta_{w} / c, \theta_{w}=0$, and $\varsigma=\Gamma_{0} /\left(\hbar \omega-\hbar \omega_{0}+\right.$ $i \gamma$ ) denotes the excitonic contribution to the scattered light in which $\omega$ and $\omega_{0}$ are frequencies of the incident light and exciton resonance, respectively.

$\gamma$ is nonradiative exciton damping rate and $\Gamma_{0}$ is radiative damping rate of a single quantum well [16]. Considering (1) total transfer matrix surrounded by other halves of barriers can be written as

$$
M_{N}^{(\alpha)}=M_{b}^{1 / 2}\left(M_{j}^{(\alpha)}\right)^{N} M_{b}^{1 / 2}
$$

Generally $M_{N}^{(\alpha)}$ is a $2 \times 2$ matrix that can be written as $M_{N}^{(\alpha)}=$ $\left(\begin{array}{ll}M_{11} & M_{12} \\ M_{21} & M_{22}\end{array}\right)$. As well as total reflectivity of the MQWs can be given by

$$
R=\left|\frac{r_{01}+r}{1+r r_{01}}\right|^{2}
$$

where $r=-M_{21} / M_{22}$ is the reflection coefficient of the QW and $r_{01}=-\left(n_{b}-1\right) /\left(n_{b}+1\right)$ is the Fresnel reflection coefficient.

By adding a defect into the midway of the MOWs, total transfer matrix can be given by (7). In this case (5) will be written as

$$
\begin{aligned}
& \widetilde{M}_{N}^{(\alpha)} \\
& \quad=M_{b}^{1 / 2}\left(M_{j}^{(\alpha)}\right)^{N / 2} M_{b}^{1 / 2} M_{D} M_{b}^{1 / 2}\left(M_{j}^{(\alpha)}\right)^{N / 2} M_{b}^{1 / 2},
\end{aligned}
$$

where

$$
M_{D}=\left(\begin{array}{cc}
e^{i \varphi_{D}} & 0 \\
0 & e^{-i \varphi_{D}}
\end{array}\right)
$$

and $\varphi_{D}=\omega n_{D} d_{D} \cos \theta_{D} / c$ (herein $\theta_{D}=0$ ). $n_{D}, d_{D}, \theta_{D}$, and $c$ are the refractive index of defect, the defect width, incident angle, and velocity of light through the vacuum, respectively.

It should be noted that

$$
\widetilde{M}_{N}^{(\alpha)}=\left(\begin{array}{ll}
\widetilde{M}_{11} & \widetilde{M}_{12} \\
\widetilde{M}_{21} & \widetilde{M}_{22}
\end{array}\right)
$$

and in this case $r$ is defined as $r=-\widetilde{M}_{21} / \widetilde{M}_{22}$. 


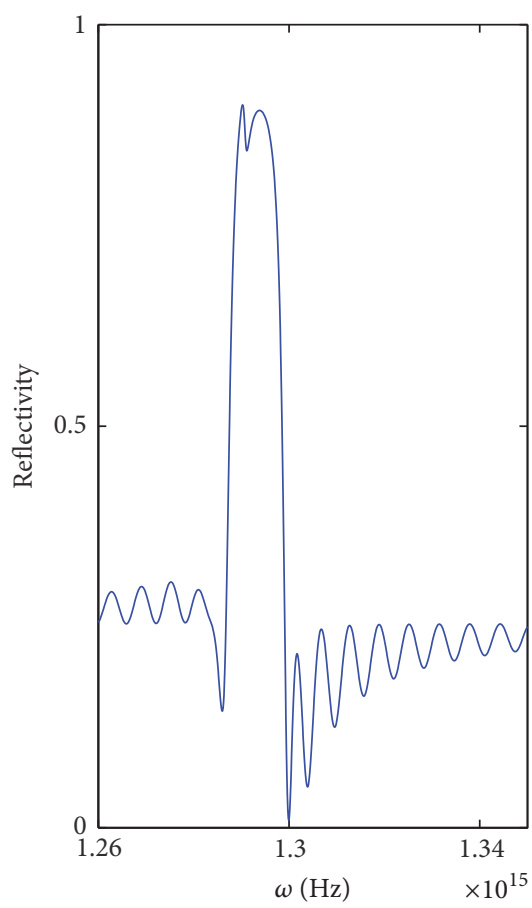

(a)

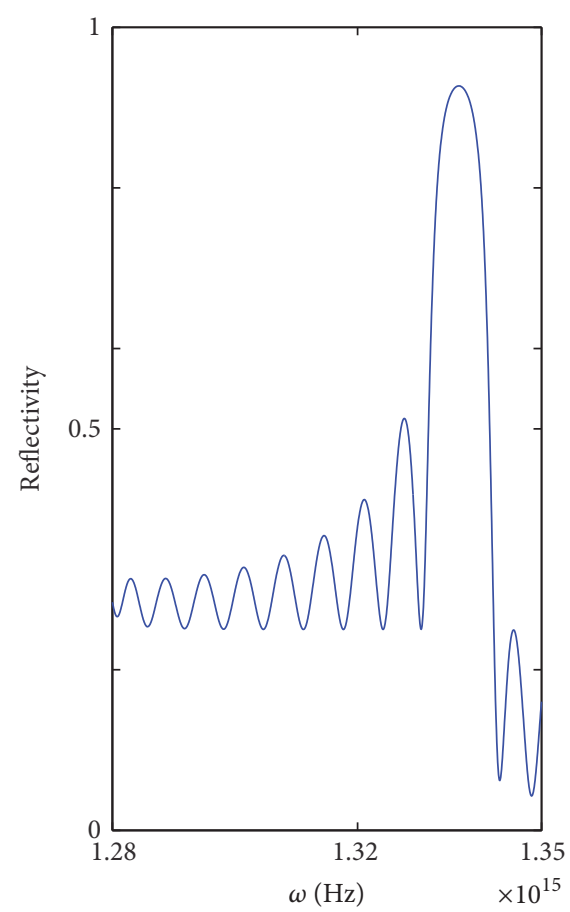

(b)

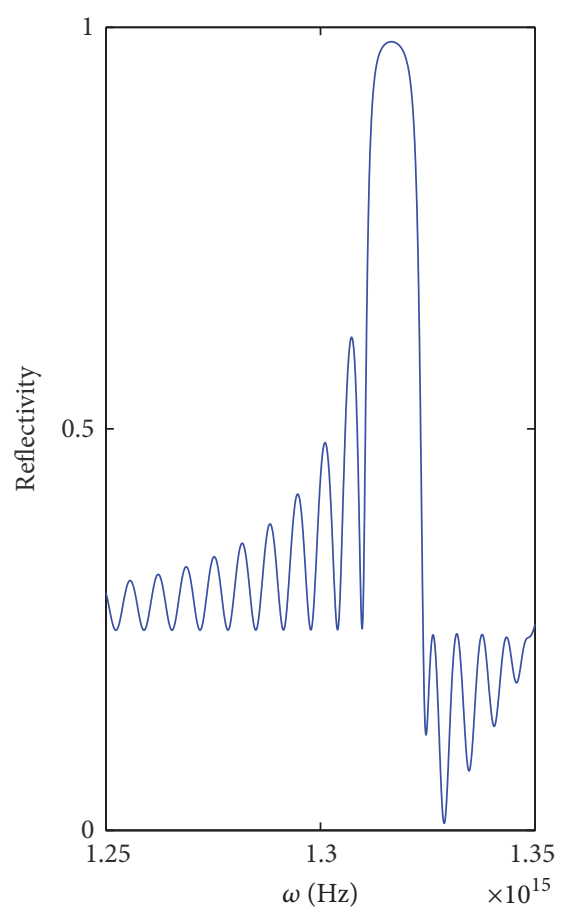

(c)

FIGURE 2: Reflectivity of 200-periodic QWs without presence of a strong pump pulse for (a) $\operatorname{In}_{x} \mathrm{Ga}_{1-x} \mathrm{As} / \mathrm{InP}$, (b) $\mathrm{In}_{x} \mathrm{Ga}{ }_{1-x} \mathrm{As} / \mathrm{GaAs}$, and (c) $\operatorname{In}_{x} \mathrm{Al}_{1-x} \mathrm{As} / \mathrm{InP}$.

\section{Results and Discussion}

In the numerical calculations performed on a MQW consisting of 200 periods of InGaAs quantum wells and InP barriers, the optical and geometrical parameters of the system at $\lambda=1.5 \mu \mathrm{m}$ were considered as follows: $n_{b}=3.03 d_{b}=$ $233.3 \mathrm{~nm}, n_{w}=3.2003$, and $d_{w}=7 \mathrm{~nm}$ which are barrier refractive index, barrier width, and quantum well refractive index and width, respectively [11, 17]. As it is known, the excitonic resonance energy at $10 \mathrm{~K}$ is $\bar{h} \omega_{0}=0.849 \mathrm{ev}$ [18]. Considering $\Gamma_{0}=27.6 \mu \mathrm{ev}$ and $\gamma=0.6 \mu \mathrm{ev}$ and (6), reflectivity of the MQW under normal incidence illumination was numerically computed and shown in Figure 2(a) [11, 12]. The same calculations were performed for two other kinds of MQWs comprising 200-periodic InAlAs/InP at $\lambda=1.9 \mathrm{~nm}$ and InGaAs $/ \mathrm{GaAs}$ at $\lambda=1.7 \mathrm{~nm}$, with the specifications of $n_{b}=3.03, d_{b}=107.7 \mathrm{~nm}, n_{w}=2.951$, and $d_{w}=7.4 \mathrm{~nm}$ with $\Gamma_{0}=31 \mu \mathrm{ev}$ and $\gamma=0.2 \mu \mathrm{ev}$ for InAlAs/InP and $n_{b}=3.365$, $d_{b}=43.2 \mathrm{~nm}, n_{w}=3.20031$, and $d_{w}=7.3 \mathrm{~nm}$ with $\Gamma_{0}=$ $37 \mu \mathrm{ev}$ and $\gamma=1.01 \mu \mathrm{ev}$ for InGaAs/GaAs $[19,20]$. The pump pulse which is chosen to be a Gaussian pulse is spectrally centered $3 \mathrm{meV}$ below the heavy-hole (hh) exciton resonance and has $1 \mathrm{ps}$ duration, with an intensity of $12 \mathrm{MW} / \mathrm{cm}^{2}$ [11].

Figures 2(a)-2(c) demonstrate that the computed reflectivity is $87 \%, 92 \%$, and $96.5 \%$ for InGaAs/InP, InGaAs/GaAs, and InAlAs/InP, respectively. These figures exhibit that the MQW made by InAlAs/InP is associated with higher reflectivity compared to the other two MQWs. To the best of our knowledge, this reflectivity is the highest value that has already been reported in literature.
Figures 3(a)-3(c) show the effect of a pump pulse on the reflectivity for the three MQWs in the presence of a strong pulse pump. It is evident that, by imposing a strong pulse pump, reflectivity is noticeably (about $40 \%$ ) reduced.

To realize the optical Stark effect (OSE), band-gaps of the considered MQWs versus the incident angle were studied with and without a strong pump. Figures 4(a)-4(c) show the band-gaps for InGaAs/GaAs, InGaAs/InP, and InAlAs/InP in the absence of the pump.

Figures 5(a)-5(c) depict the same band-gaps for InGaAs/GaAs, InGaAs/InP, and InAlAs/InP in the presence of the pump.

By applying strong external pump pulse with a frequency close to the excitonic frequency, the photonic band-gaps are modulated and for a given frequency the reflectivity became out of resonance state as illustrated in Figures 5(a)-5(c).

Therefore, the system can act as an all-optical switch, considering a significant shift in optical gap-band of the photonic crystal. Further investigation was performed to evaluate the impact of the incident angle on the reflectivity. Equation (6) was used to plot reflectivity under three different incident angles for InGaAs/InP MQW (Figure 6(a)). As can be seen in Figure 6 without external pump the reflection peak of InGaAs/InP is complete at the resonant frequency for $\theta=0^{\circ}$. However, evident shifts along with little suppression can be seen in reflectivity by changing the incident angle to $\theta=30^{\circ}$ and $\theta=60^{\circ}$.

The effect of incident angle and width error was also studied for the other two MQWs shown in Figures 7 and 8 . As it was expected we can see a shift in the reflectivity by 


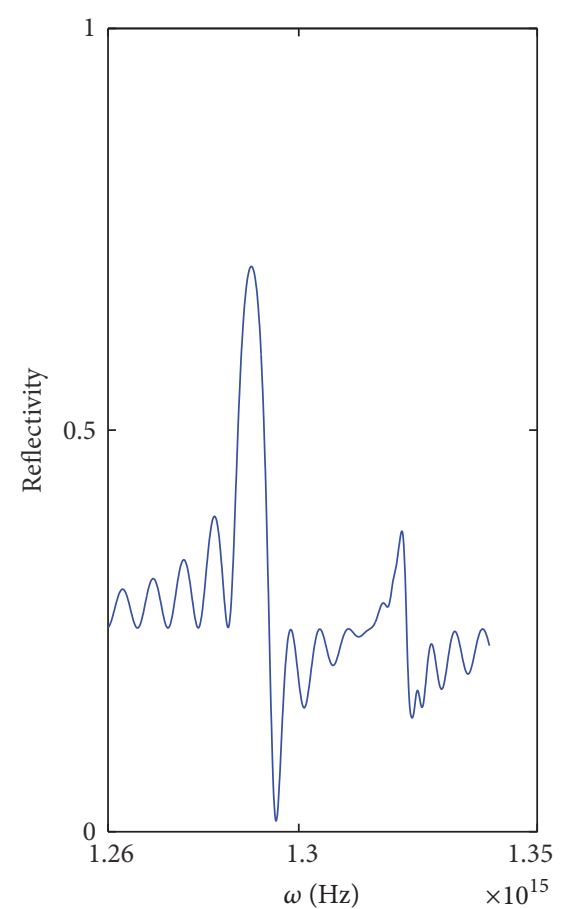

(a)

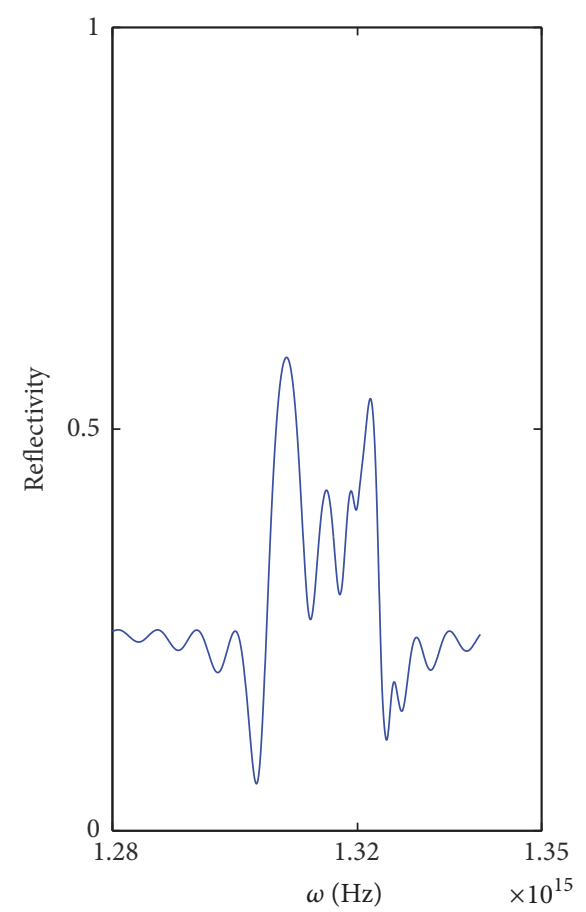

(b)

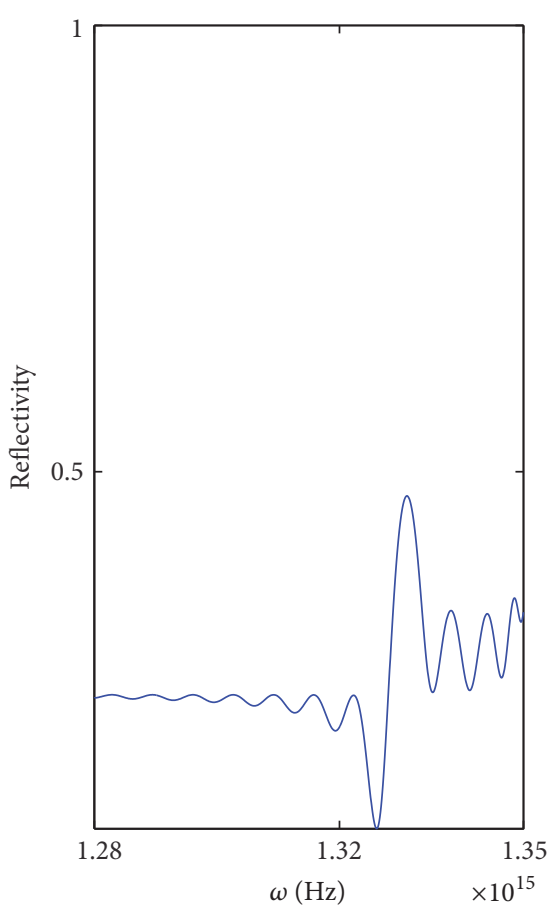

(c)

FIGURE 3: Reflectivity of 200-periodic QWs with presence of a strong pump pulse for (a) $\operatorname{In}_{x} \mathrm{Ga}_{1-x} \mathrm{As} / \mathrm{InP}$, (b) $\mathrm{In}_{x} \mathrm{Ga} \mathrm{1}_{1-x} \mathrm{As} / \mathrm{GaAs}$, and (c) $\operatorname{In}_{x} \mathrm{Al}_{1-x} \mathrm{As} / \mathrm{InP}$.

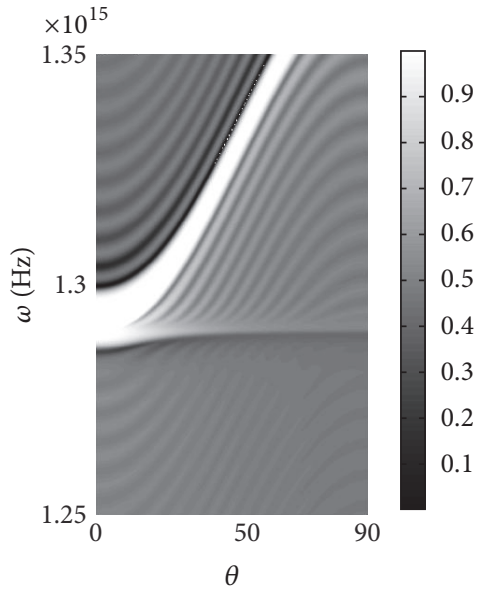

(a)

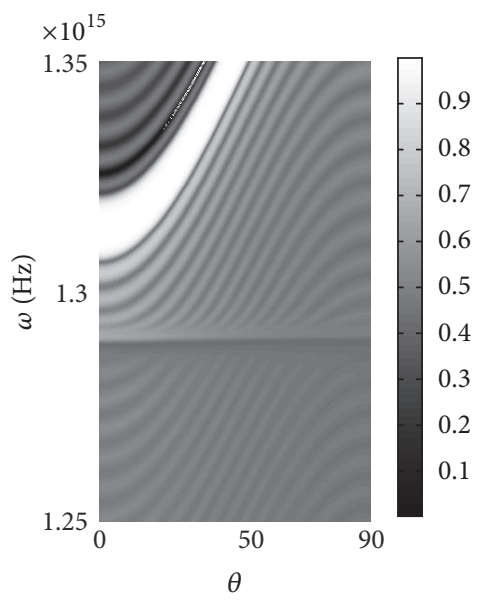

(b)

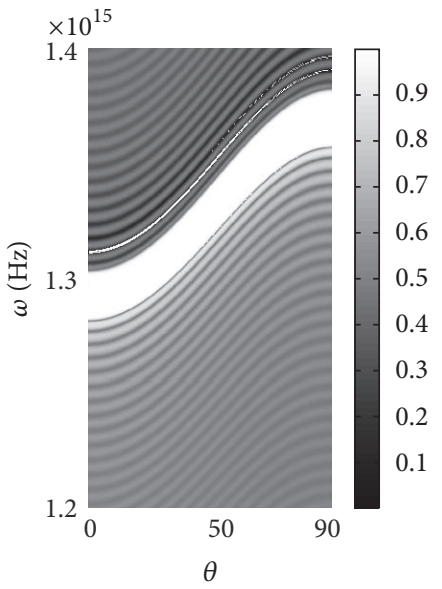

(c)

Figure 4: Black areas are band-gaps of photonic crystals containing 200-periodic (a) InGaAs/InP, (b) InGaAs/GaAs, and (c) InAlAs/InP QWs without implementing an external pump pulse.

any change in the incident angle for both of InGaAs/GaAs (Figure 7(a)) and InAlAs/InP (Figure 8(a)) as well.

As it can be seen in Figures 7(b) and 8(b), considering $1 \mathrm{~nm}$ and $2 \mathrm{~nm}$ width errors for both of InGaAs/GaAs and InAlAs/InP MWQs were associated with slight suppression in reflectivity.

We further studied the impact of two types of defects on the reflectivity of InGaAs/InP MQW by adding a glass layer and doubling the width of central well. Adding a glass layer of thickness $d_{g}=13.16 \mathrm{~nm}$ and refractive index $n_{g}=$ 1.6 resulted in reduction of reflectivity at the resonance (Figure 9(b)). Furthermore, doubling the width of the central well was associated with a drop of reflectivity at the resonance (Figure 9(c)). Eventually, it is worth noting that implementing any defect into MQW which alters the Bragg condition causes reduction of reflectivity performance. 


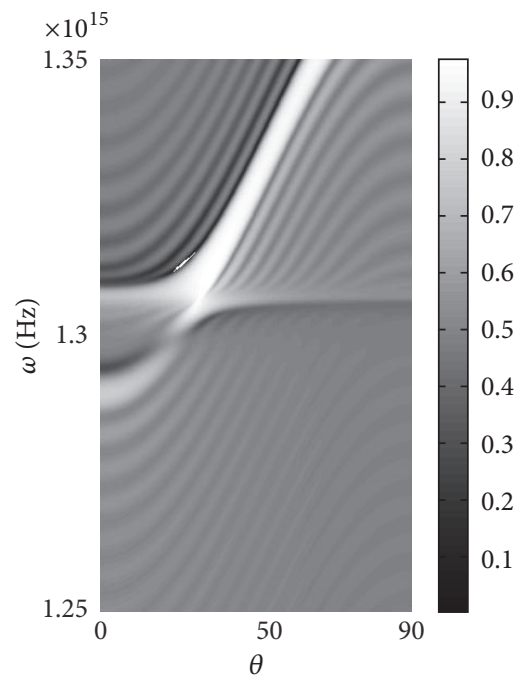

(a)

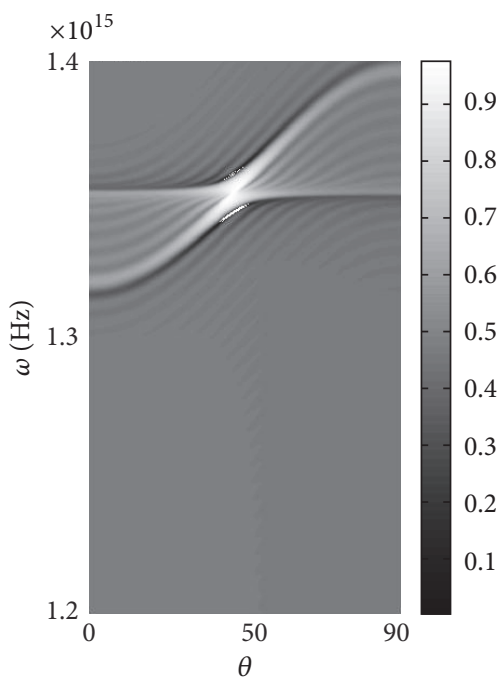

(b)

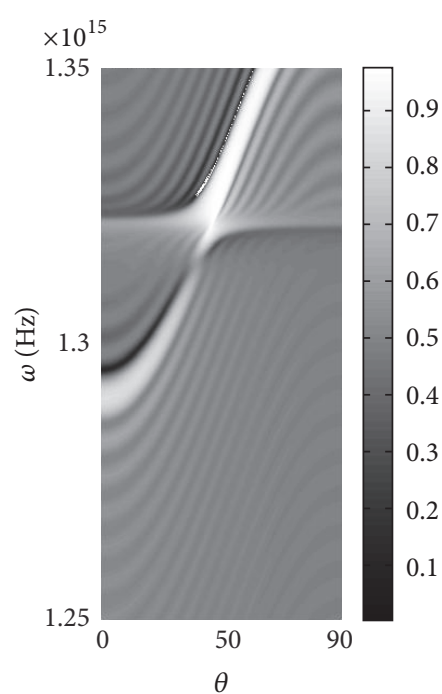

(c)

FIGURE 5: Black areas are band-gaps of photonic crystals containing 200-periodic (a) InGaAs/InP, (b) InGaAs/GaAs, and (c) InAlAs/InP QWs with implementing an external pump pulse.

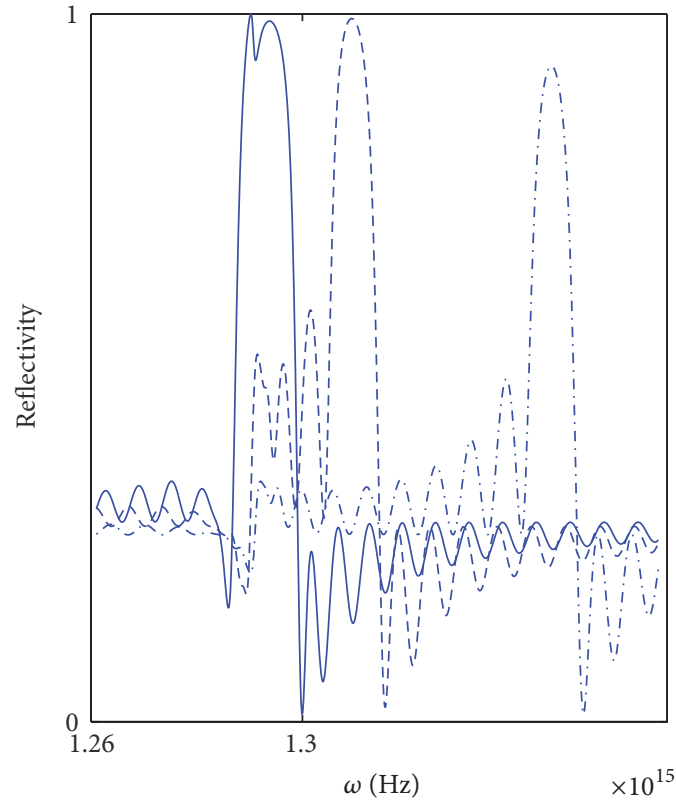

(a)

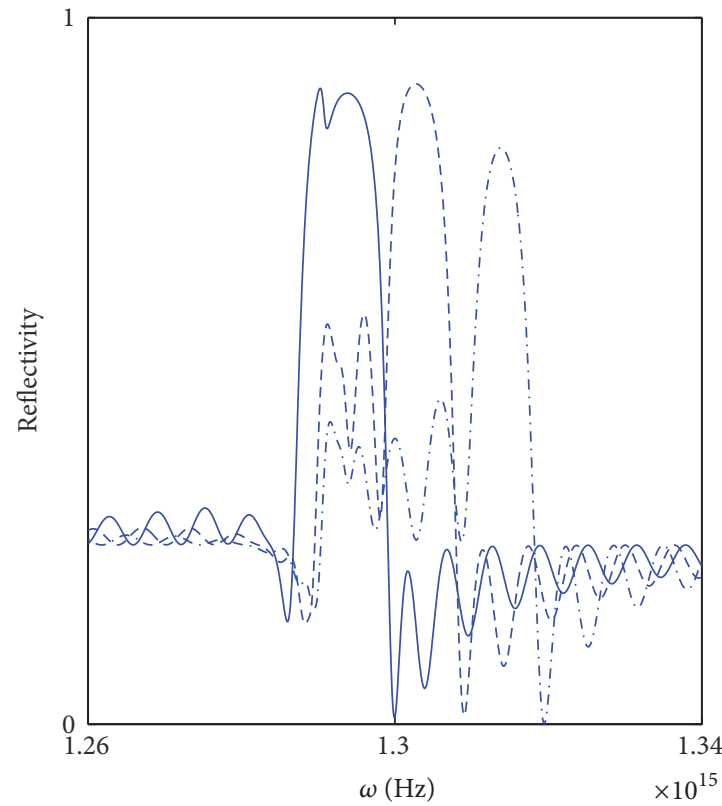

(b)

Figure 6: (a) Reflectivity of 200-periodic InGaAs/InP quantum well for $\theta=0^{\circ}$ (solid line), $\theta=30^{\circ}$ (dashed line), and $\theta=60^{\circ}$ (dashed-dot line). (b) Effect of width error in the switch performance. The solid line shows the reflectivity with no error. The dashed line shows $1 \mathrm{~nm}$ error of both quantum barrier and well width on the reflectivity. Dashed-dot line shows $2 \mathrm{~nm}$ error of quantum barrier and well width on the reflectivity. (b) illustrates the effect of $1 \mathrm{~nm}$ and $2 \mathrm{~nm}$ width errors showed by dashed and dashed-dotted lines, respectively, on the reflectivity of InGaAs/InP MQW in normal incident. Resultantly, we see that even $1 \mathrm{~nm}$ width error causes a shift from resonance frequency and the reflection will also be suppressed slightly. Besides, $2 \mathrm{~nm}$ width error on the quantum barrier and well width results in a significant impact on the switch performance and the system will completely be suppressed. 


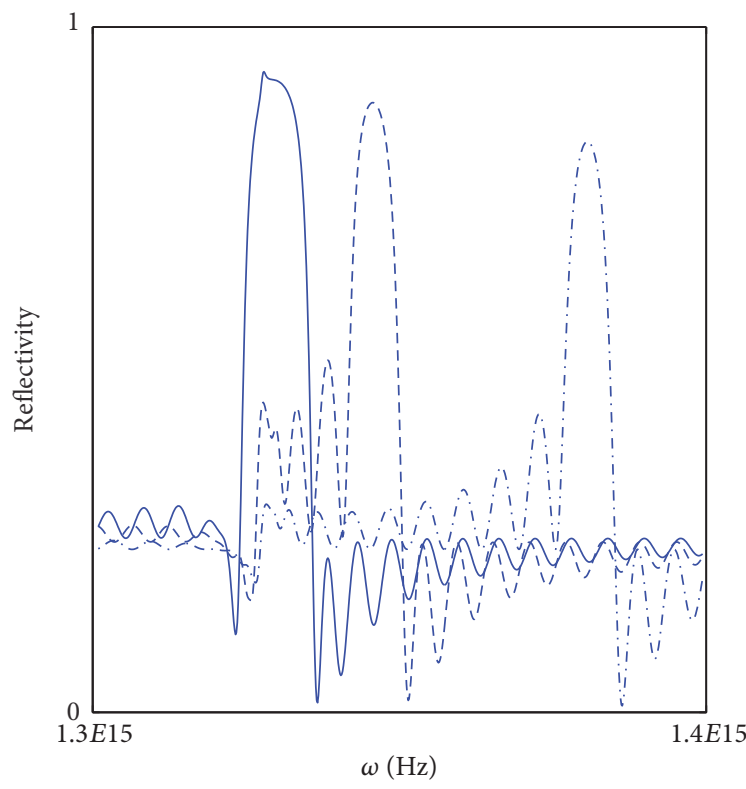

(a)

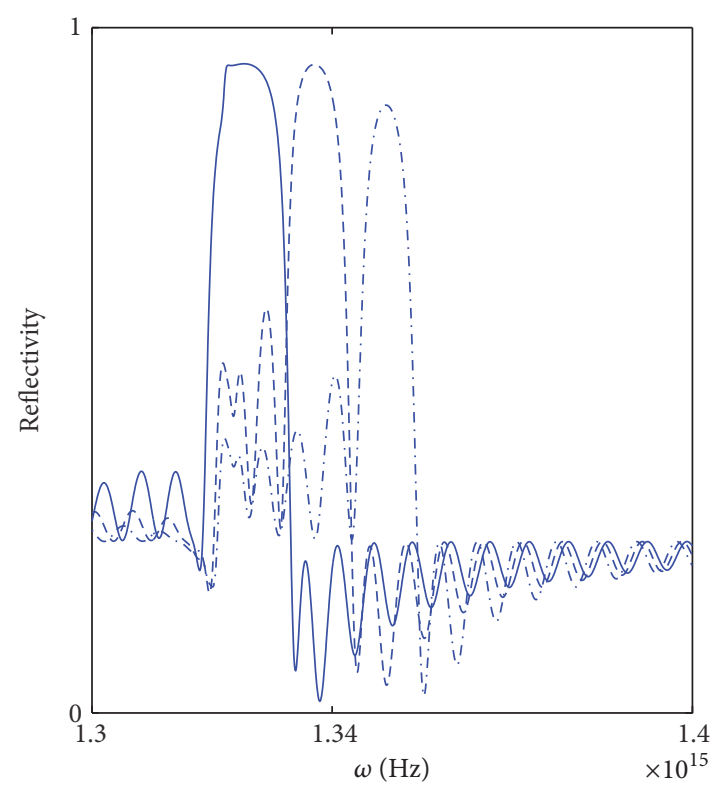

(b)

Figure 7: (a) Reflectivity of 200-periodic InGaAs/GaAs quantum well for $\theta=0^{\circ}$ (solid line), $\theta=30^{\circ}$ (dashed line), and $\theta=60^{\circ}$ (dashed-dot line). (b) Effect of width error on the switch performance. Solid line shows the reflectivity with no error. Dashed line shows 1 nm error of both quantum barrier and well width on the reflectivity. Dashed-dot line shows $2 \mathrm{~nm}$ error of quantum barrier and well width on the reflectivity.

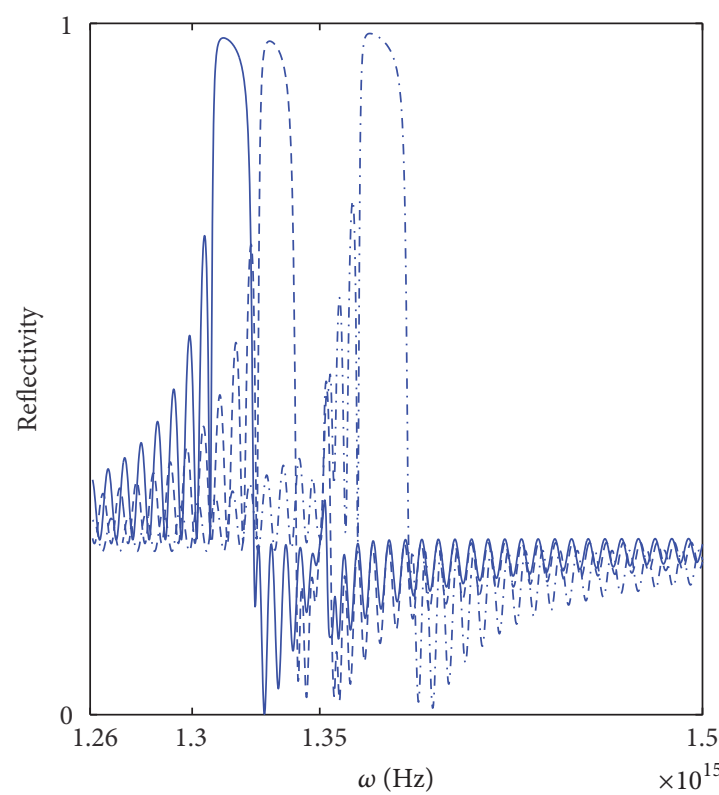

(a)

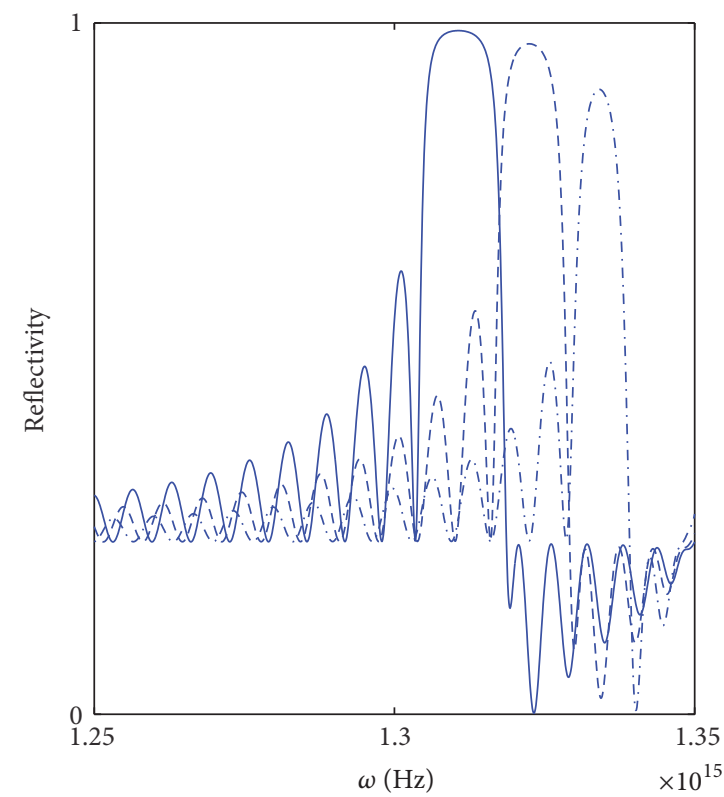

(b)

Figure 8: (a) Reflectivity of 200-periodic InAlAs/InP quantum well for $\theta=0^{\circ}$ (solid line), $\theta=30^{\circ}$ (dashed line), and $\theta=60^{\circ}$ (dashed-dot line). (b) Effect of width error on the switch performance. Solid line shows the reflectivity with no error. Dashed line shows $1 \mathrm{~nm}$ error of both quantum barrier and well width on the reflectivity. Dashed-dot line shows $2 \mathrm{~nm}$ error of quantum barrier and well width on the reflectivity.

\section{Conclusion and Outlook}

This study demonstrated a theoretical model of all-optical switches based on $\operatorname{In}_{x} \mathrm{Ga}_{1-x} \mathrm{As} / \mathrm{InP}, \mathrm{In}_{-x} \mathrm{Ga}_{1-x} \mathrm{As} / \mathrm{GaAs}$, and $\mathrm{In}_{x} \mathrm{Al}_{1-x} \mathrm{As} / \mathrm{InP}$ BSQWs structures and evaluated their performances with and without Stark effect. By means of comparisons, it was found that the switch adopting $\mathrm{In}_{x} \mathrm{Al}_{1-x} \mathrm{As} / \mathrm{InP} \mathrm{BSQWs}$ structure has the maximum reflectivity and the employment of the Stark effect mechanism for excitonic levels can be utilized to control the crystal band-gap. 


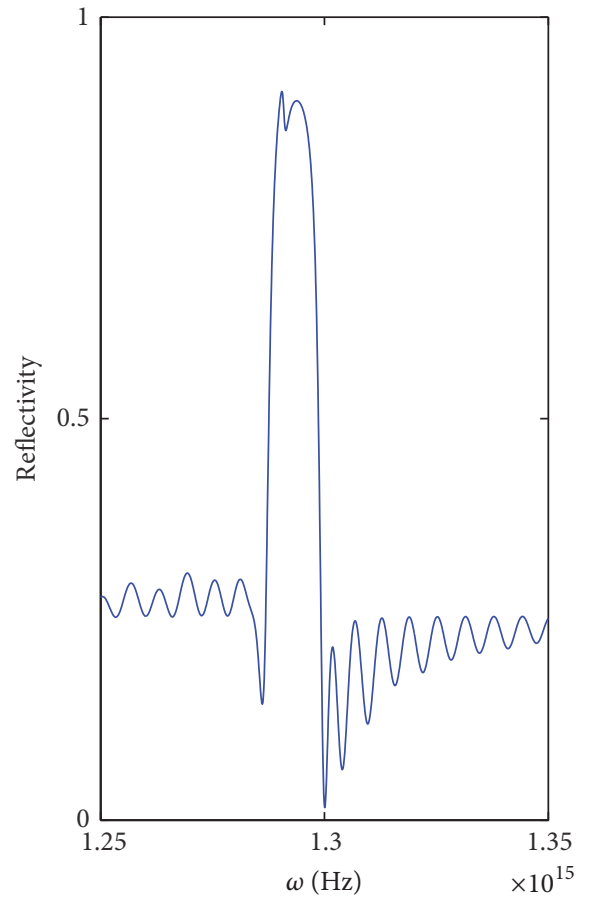

(a)

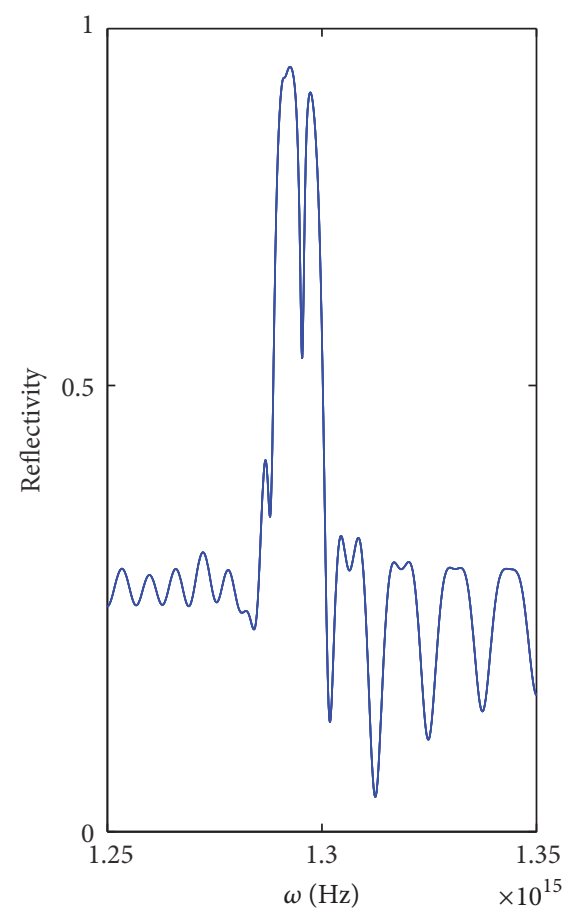

(b)

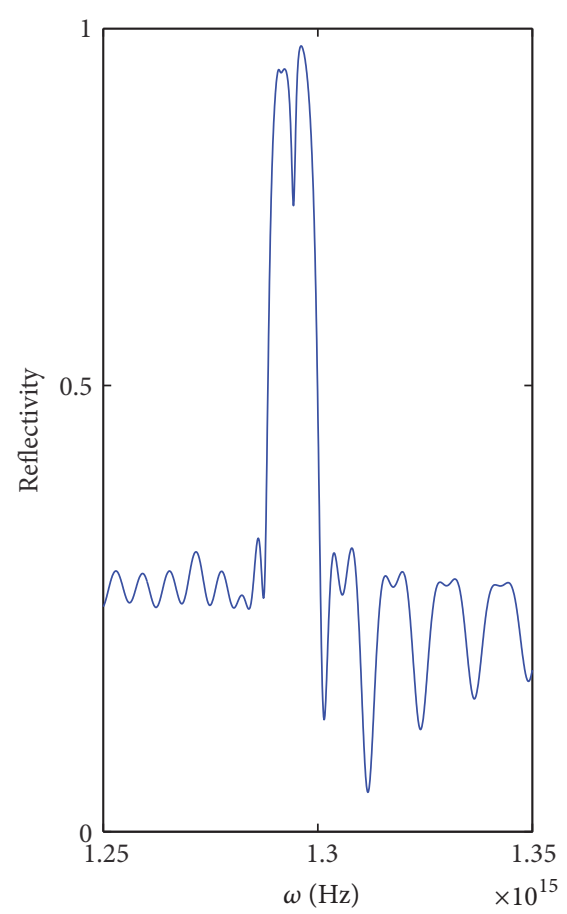

(c)

FIGURE 9: Reflectivity spectra of InGaAs/InP QMW (a) without any defect, (b) with inserting a glass layer as a defect with refractive index $n_{g}=1.6$ and thickness of $d_{g}=13.16 \mathrm{~nm}$, and (c) with doubling the width of the central well.

All these BSQWs, specially $\operatorname{In}_{x} \mathrm{Al}_{1-x} \mathrm{As} / \mathrm{InP}$, are appropriate for using as all-optical switches. The influence of width error was also studied. It was found that any error even $1 \mathrm{~nm}$ or any defect can affect the Bragg condition and cause reduction of reflectivity performance and consequently impact on the switch performance.

\section{Conflicts of Interest}

The authors declare that there are no conflicts of interest regarding the publication of this paper.

\section{Acknowledgments}

The authors thank the Physics Department in the University of Tabriz for the use of their equipment.

\section{References}

[1] J. D. Joannapoulos, S. G. Johnson, J. N. Winn, and Meude R. D., Photonic Crystals: Modeling the Flow of Light, Princeton, Mo, USA, Princeton University Press, 2011.

[2] D. N. Chigrin, A. V. Lavrinenko, D. A. Yarotsky, and S. V. Gaponenko, "Observation of total omnidirectional reflection from a one-dimensional dielectric lattice," Applied Physics A: Materials Science and Processing, vol. 68, no. 1, pp. 25-28, 1999.

[3] S. John, "Strong localization of photons in certain disordered dielectric superlattices," Physical Review Letter, vol. 58, article 2486, 1987.
[4] S. John and T. Quang, "Spontaneous emission near the edge of a photonic band gap," Physical Review A, vol. 50, no. 2, pp. 1764$1769,1994$.

[5] P. S. J. Russell, S. Tredwell, and P. J. Roberts, "Full photonic bandgaps and spontaneous emission control in $1 \mathrm{D}$ multilayer dielectric structures," Optics Communications, vol. 160, no. 1-3, pp. 66-71, 1999.

[6] P. Kramper, M. Agio, C. M. Soukoulis et al., "Highly directional emission from photonic crystal waveguides of subwavelength width," Physical Review Letters, vol. 92, no. 11, Article ID 113903, 2004.

[7] B. Schulkin, L. Sztancsik, and J. F. Federici, "Analytical solution for photonic band-gap crystals using Drude conductivity," American Journal of Physics, vol. 72, no. 8, pp. 1051-1054, 2004.

[8] D. Soto-Puebla, M. Xiao, and F. Ramos-Mendieta, "Optical properties of a dielectric-metallic superlattice: the complex photonic bands," Physics Letters, Section A: General, Atomic and Solid State Physics, vol. 326, no. 3-4, pp. 273-280, 2004.

[9] S. Feng, J. Merle Elson, and L. Overfelt, "Optical properties of multilayer metal-dielectric nanofilms with all-evanescent modes," Optics Express, vol. 13, no. 11, pp. 4113-4124, 2005.

[10] D. Bria, B. Djafari-Rouhani, A. Akjouj et al., "Band structure and omnidirectional photonic band gap in lamellar structures with left-handed materials," Physical Review E-Statistical, Nonlinear, and Soft Matter Physics, vol. 69, no. 6, Article ID 066613, 2004.

[11] W. Tao, L. Gang, and Z. Chen, "The theoretical investigation of all-optical polarization switching based on InGaAs(P) Braggspaced quantum wells," Optics Express, vol. 16, no. 1, pp. 127-132, 2008. 
[12] W. Tao and L. Qing, "Ultrafast polarization optical switch constructed from one-dimensional photonic crystal and its performance analysis," Chinese Science Bulletin, vol. 54, no. 20, pp. 3663-3669, 2009.

[13] W. J. Johnson and J. P. Prineas, "Ultrafast all-optical polarization switching in Bragg-spaced quantum wells at $80 \mathrm{~K}$," Journal of Applied Physics, vol. 101, no. 4, Article ID 046101, 2007.

[14] J. P. Prineas and J. Y. Zhou, "Ultrafast ac Stark effect switching of the active photonic band gap from Bragg-periodic semiconductor quantum wells," Applied Physics Letters, vol. 81, no. 23, pp. 4332-4334, 2002.

[15] M. V. Erementchouk and L. I. Deych, "Optical properties of onedimensional photonic crystals based on multiple-quantum-well structures," Physical Review B, vol. 71, no. 23, Article ID 235335, 2005.

[16] J. P. Prineas and C. Cao, "Resonant photonic band gap structures realized from molecular-beam-epitaxially grown InGaAs/GaAs Bragg-spaced quantum wells," Journal of Applied Physics, vol. 100, no. 6, Article ID 063101, 2006.

[17] S. Adachi, "Lattice thermal conductivity of group-IV and III-V semiconductor alloys," Journal of Applied Physics Letters, vol. 102, no. 6, Article ID 063502, 2007.

[18] S. Adachi, "Optical dispersion relations for GaP, GaAs, GaSb, InP, InAs, InSb, $\mathrm{Al}_{x} \mathrm{Ga}_{1-x} \mathrm{As}$, and $\mathrm{In}_{1-x} \mathrm{Ga}_{x} \mathrm{As}_{y} \mathrm{P}_{1-y}$, Journal of Applied Physics, vol. 66, no. 4, 1989.

[19] C. Ghidoni, R. Magri, and S. Ossicini, The electric and optical properties of InGaAs/InP and InAlAs/InP superlattices, vol. 2131, Elsevier Science, Amsterdam, Netherlands, 2001.

[20] S. Adachi, "Material parameters of $\mathrm{In}_{1-x} \mathrm{Ga}_{x} \mathrm{As}_{y} \mathrm{P}_{1-y}$ and related binaries," Journal of Applied Physics, vol. 53, no. 12, pp. 87758792, 1982. 

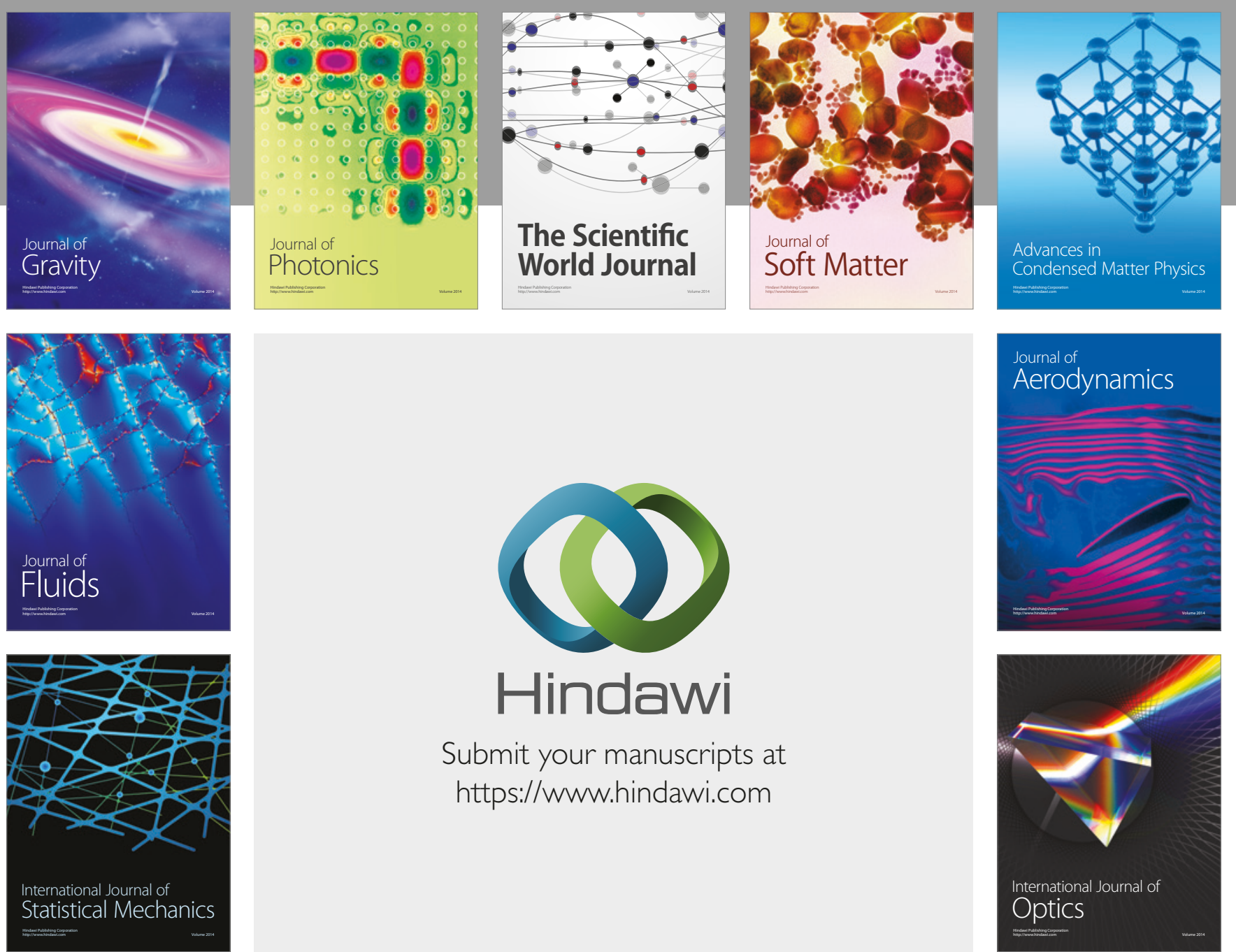

Submit your manuscripts at

https://www.hindawi.com
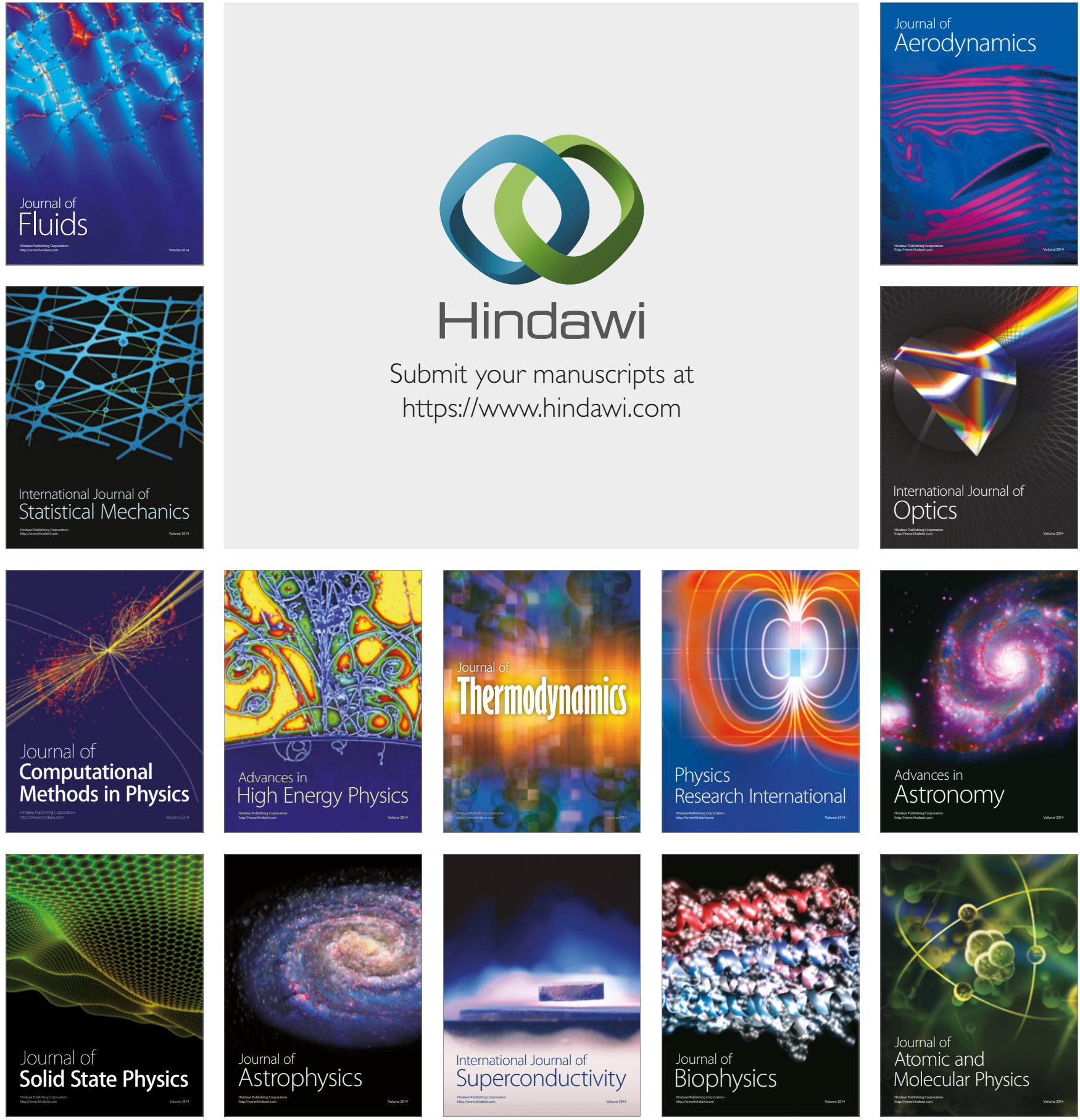